\title{
Conecta-SUS: o uso das redes sociais na divulgação de informações de ações e serviços do Sistema Único de Saúde
}

\author{
Conecta-SUS: the use of social networks in the dissemination of information on actions and services \\ of the Unified Health
}

Conecta-SUS: el uso de las redes sociales en la difusión de información sobre acciones y servicios del Sistema Unificado de Salud

\section{Resumo}

O objetivo desse estudo é discutir o impacto do projeto Conecta-SUS sobre o Sistema Único de Saúde, enquanto uma estratégia de educação em saúde, através dos perfis das redes sociais utilizando as tecnologias de informação e comunicação: Instagram $^{\circledR}$, Facebook $^{\circledR}$ e Twitter $^{\circledR}$. A Lei Orgânica da Saúde sancionada em 1990, regula as ações e serviços de saúde em todo o território nacional e estabelece, princípios, diretrizes e objetivos do Sistema Único de Saúde, mencionando que o cidadão tem direito a ter conhecimento sobre o funcionamento desse sistema, por meio da divulgação de informações quanto ao potencial dos serviços de saúde e sua utilização pelo usuário. O Conecta-SUS é um projeto de extensão do Curso de Medicina da Universidade Federal de Juiz de Fora, Governador Valadares, com a participação interdisciplinar de discentes e docentes dos cursos de saúde. Foram criadas contas nestas redes sociais, administradas pelo professor coordenador e por um aluno do projeto. As publicações foram divididas em três padrões de conteúdo: teóricas, práticas e referentes à Covid-19. Foram realizadas 136 publicações: 30 teóricas, 44 práticas, 62 referentes à Covid-19. As publicações receberam 5230 curtidas, sendo 4694 no Instagram $^{\circledR}, 355$ no Facebook $^{\circledR}, 181$ no Twitter $^{\circledR}$. Logo, a construção digital de saberes tem impacto relevante, tanto para professores, alunos e população 
geral, pois permite que esta tenha acesso de forma rápida e segura, numa linguagem simples, a várias informações, muitas vezes, desconhecidas ou até mesmo não compreendidas sobre o Sistema Único de Saúde.

Palavras-chave: Sistema Único de Saúde; Rede social; Saúde pública; Tecnologias de informação; Comunicação.

\begin{abstract}
The objective of this study is to discuss the impact of the Conecta-SUS project as a health education strategy on the Unified Health System, through the profiles of social networks using information and communication technology: Instagram ${ }^{\circledR}$, Facebook ${ }^{\circledR}$ and Twitter ${ }^{\circledR}$. The Organic Health Law enacted in 1990, regulates health actions and services throughout the national territory and establishes principles, guidelines and objectives of the Unified Health System, mentioning that the citizen has the right to know about the functioning of this system, through the dissemination of information about the potential of health services and their use by the user. Conecta-SUS is an extension project of the Medicine Course at the Federal University of Juiz de Fora, Governador Valadares, with the interdisciplinary participation of students and professors of health courses. Accounts were created on these social networks, managed by the coordinating professor and a student of the project. The publications were divided into three content standards: theoretical, practical and referring to Covid-19. 136 publications were made: 30 theoretical, 44 practical, 62 referring to Covid-19. The publications received 5230 likes, 4694 on Instagram $®, 355$ on Facebook®, 181 on Twitter Therefore, the digital construction of knowledge has a relevant impact, both for teachers, students and the general population, as it allows them to have quick and secure access, in simple language, to various information, often unknown or even not understood about the Health Unic System.
\end{abstract}

Keywords: Unified Health System; Social Network; Public health; Information technologies; Communication.

\title{
Resumen
}

El objetivo de este estudio es discutir el impacto del proyecto Conecta-SUS enquanto una estrategia de educación en salud sobre el Sistema Único de Saúde, através dos perfis das redes sociales utilizando como tecnología de información y comunicación: Instagram ${ }^{\circledR}$, Facebook ${ }^{\circledR}$ y Twitter®. La Ley Orgánica de Salud promulgada en 1990, regula las acciones y servicios de salud en todo el territorio nacional y establece principios, lineamientos y objetivos del Sistema Único de Salud, mencionando que el ciudadano tiene derecho a conocer el funcionamiento de este sistema, a través de la difusión de información sobre el potencial de los servicios de salud y su uso por parte del usuario. Conecta-SUS es un proyecto de extensión del Curso de Medicina de la Universidad Federal de Juiz de Fora, Governador Valadares, con la participación interdisciplinaria de estudiantes y profesores de cursos de salud. Se crearon cuentas en estas redes sociales, gestionadas por el profesor coordinador y un alumno del proyecto. Las publicaciones se dividieron en tres estándares de contenido: teórico, práctico y referido a Covid-19. Se realizaron 136 publicaciones: 30 teóricas, 44 prácticas, 62 referidas al Covid-19. Las publicaciones recibieron 5230 me gusta, 4694 en Instagram ${ }^{\circledR}, 355$ en Facebook ${ }^{\circledR}, 181$ en Twitter ${ }^{\circledR}$. Logo, a construção digital de saberes tem impacto relevante, tanto para professores, alunos e população geral pois permite que esta tenha acesso de forma rápida e segura, numa linguagem simples, a várias informações, muitas vezes, desconhecidas ou até mesmo não compreendidas sobre o Sistema Único de Salud. Palabras clave: Sistema Único de Salud; Red social; Salud pública; Tecnologías de la información; Comunicación.

\section{Introdução}

O Conecta-SUS é um projeto de extensão do Curso de Medicina da Universidade Federal de Juiz de Fora (UFJF) Campus Governador Valadares, aprovado em 2019, com a participação interdisciplinar de cursos da área da saúde, com discentes e docentes da medicina, fisioterapia, farmácia e nutrição. O principal objetivo do projeto consiste na divulgação e promoção por meio das redes sociais dos serviços oferecidos e garantidos pelo sistema público de saúde brasileiro, o Sistema Único de Saúde (SUS).

O SUS é regido pela Lei 8080, Lei Orgânica da Saúde, que dispõe como princípio organizativo - "o direito ao acesso à informação referente aos serviços de saúde e sua utilização pelo usuário", descrito no capítulo 2 , artigo $7^{\circ}$, inciso $6^{\circ}$ (Brasil, 1990, sp). Nesse sentido, se manifesta a necessidade e a relevância da utilização de dispositivos e formas de capilarização das inúmeras informações, normativas, atualizações, campanhas, direitos, deveres, acesso relacionados ao cidadão e ao SUS, viabilizando esse conjunto de conhecimentos por meio de mecanismos tecnológicos, como as redes sociais, apoiando, ampliando e fomentando o acesso à informação ao maior número possível de pessoas e consequentemente da sociedade.

Nas sociedades, grande parte da população está conectada às mídias sociais, dessa forma, elas estão se tornando uma ampla ferramenta de divulgação de conhecimento científico rápida e prática. O ambiente virtual permite que diversos assuntos sejam pesquisados, já que há uma enorme quantidade de conteúdos publicados. Entretanto, é necessário ter cautela na obtenção 
dessas informações, tendo em vista que muitas ferem o quesito qualidade, estão sujeitas a diversos vieses e, se tratando da área da saúde, a qualidade da informação é bastante importante (Moretti, Oliveira \& Silva, 2012).

O Conecta-SUS, tem o propósito de dar continuidade às ações realizadas desde a sua criação, estando atualmente em andamento, cumprindo um papel oportuno de fomento e expansão, sobretudo de colaboração diante os preceitos legais, programas e políticas públicas de saúde e as garantias do SUS aos usuários. Posto a normativa supracitada, se torna imperativo a criação de espaços de divulgação e de amplo alcance para o compartilhamento de dados, documentos e informações por vezes restrito à diversos meios. Além disso, essa proposta permite compartilhar informações sobre saúde pública, contribuindo para a formação de cidadãos mais conscientes, favorecendo o protagonismo das pessoas acerca de seu processo saúde/doença e sobre o direito à saúde, que, no Brasil, é constitucional, bem como, a divulgação da rede de ações e serviços de saúde pública e seus correspondentes.

O projeto em destaque busca fornecer informações de qualidade e de maneira sistemática à toda população utilizando as tecnologias de informação e comunicação (TIC). As TIC compreendem as tecnologias de comunicação pelo computador, como acesso à internet e o uso de aplicativos e redes sociais - todas as formas virtuais de interação, relacionamentos e colaboração que se utilizam da internet como veículo para a criação de comunicação, assim como sistemas eletrônicos para registro de dados e informações (Brasil, 2018; Marteleto, 2010). Elas favorecem e transformam de maneira positiva os processos de trabalho no SUS, pois são apontadas como facilitadoras de aprendizagem e multiplicadoras do ensino (Barbosa, 2014), contribuindo para promoção da saúde, prevenção de doenças, diagnóstico e tratamento e reabilitação dos pacientes (OMS, 2017).

Sendo assim, o objetivo deste estudo é discutir o impacto do projeto Conecta-SUS sobre o SUS, enquanto uma estratégia de educação em saúde, através dos perfis das redes sociais utilizando as TIC: Instagram $^{\circledR}$, Facebook $^{\circledR}$ e Twitter $^{\circledR}$.

\section{Metodologia}

O presente estudo se caracteriza por ser um relato de experiência, que tem a finalidade de descrever a vivência de alunos e professores no Projeto Conecta-SUS, do Curso de Medicina da UFJF, campus Governador Valadares, no período compreendido entre julho de 2020 a junho de 2021. A intenção desse projeto é contribuir com a construção de conhecimento na atuação dos discentes dos cursos da área de saúde, como medicina, fisioterapia, farmácia e nutrição pertencentes a diferentes períodos da graduação, desenvolvendo a compreensão sobre os conteúdos relacionados aos cenários do SUS, ao acesso, as políticas públicas de saúde e às ações e serviços ofertados.

Inicialmente, foram criadas contas nas redes sociais Instagram $^{\circledR}$, Facebook $^{\circledR}$ e Twitter $^{\circledR}$, permitindo o acesso a informações sobre a utilização e o funcionamento dos serviços oferecidos pelo SUS. Os perfis receberam o nome de ConectaSUS (@conectasus), sendo administrados pelo professor coordenador e por um aluno do projeto, que atuava na função de preceptor do grupo de alunos que produziam as matérias, denominado de grupo de produção.

As atividades foram elaboradas pelos alunos, sob a supervisão das professoras coordenadora e colaboradora, sendo postadas, semanalmente, em dois dias alternados, pelo discente preceptor, que ficou responsável pela administração das redes. Todas as informações disponibilizadas nas publicações são baseadas em artigos científicos nacionais e internacionais, manuais, protocolos, leis, portarias e informações nacionais e regionais com fonte de referência oficiais de órgãos governamentais, as quais permitem que as matérias sejam elaboradas com conteúdo de qualidade e seguro. Todas elas são disponibilizadas na descrição das postagens com link de acesso ou referenciadas de forma a facilitar a pesquisa e conferência, caso o seguidor tenha interesse em se aprofundar mais nos assuntos ou simplesmente assegurar a veracidade das informações.

As publicações foram divididas em três modelos de padrões de conteúdo: teóricas (com foco em levar informações sobre leis, princípios, diretrizes, estrutura e história do SUS, de forma acessível para a população), práticas (dando ênfase à informações sobre ações e serviços do SUS e como acessá-los) e, por fim, trazendo o tema da pandemia da Covid-19 (criadas 
com o objetivo de levar informações de forma simples e seguras sobre temas recorrentes e variados, diante da realidade vivida no último ano).

As publicações foram feitas por plataforma de design gráfico Canva $^{\circledR}$, aplicativo popular na área de edição de postagens, o qual permite a utilização de diversas ferramentas de edição de forma gratuita e acessível em computador ou smartphone compatível. Foram utilizados computadores, notebooks ou smartphones com capacidade de memória que permitissem a correta execução dos trabalhos.

Para permitir uma melhor integração entre os participantes, uma maior coesão na construção dos conteúdos, bem como orientar e revisar as informações selecionadas para as matérias a serem publicadas, foram realizadas reuniões semanais em dia e horário pré-determinado com cronograma temático estabelecido e presença de toda a equipe do projeto, composta por dois professores e seis alunos, por meio da plataforma Google Meet ${ }^{\circledR}$.

\section{Resultados e Discussão}

As TIC Instagram ${ }^{\circledR}$, Facebook $^{\circledR}$ e Twitter $^{\circledR}$ favoreceram a expansão e conectaram o público de um modo geral com um conjunto robusto de dados, dando aos usuários da internet acesso instantâneo, conveniente às informações com assuntos específicos. No tocante ao número de seguidores, o Conecta-SUS teve de julho até dezembro de 2020, 650 seguidores e de janeiro a junho de 2021, 807 seguidores, sendo o crescimento atual estável, em aproximadamente 4\% ao mês. Desses 807 seguidores, 509 seguem a página do Instagram $^{\circledR}, 236$ do Facebook $^{\circledR}$ e 62 do Twitter $^{\circledR}$.

Devido ao fato de o acesso à internet ser popular, as redes sociais são as principais plataformas em número de usuários. O Facebook ${ }^{\circledR}$, teria, segundo estimativas de 2020 cerca de 2,5 bilhões de usuários e o Twitter $^{\circledR}{ }^{\circledR}$ cerca de 386 milhões de usuários ativos, com quase 14,5 milhões de usuários no Brasil (Xavier et al, 2020).

Nos seis primeiros meses de vigor desse projeto, obteve-se uma média de 25 curtidas por publicação no Instagram ${ }^{\circledR}$. No período de janeiro a junho de 2021, apurou-se uma média de 35 curtidas por publicação, ou seja, um aumento de $40 \%$ desse parâmetro de avaliação. A página no Instagram $^{\circledR}$ possui um alcance médio de 400 contas ao mês, sendo aproximadamente $20 \%$ dessas não seguidoras do projeto, com uma média de 386 interações com o conteúdo mensalmente. Quanto à média de impressões mensais, são cerca de 3000, sendo 3089 no mês de junho de 2021.

No Instagram $^{\circledR}$, a maioria dos dados estatísticos pertinentes utilizados para avaliar o alcance e o engajamento são fornecidos pelo Instagram Insights ${ }^{\circledR}$, que é uma ferramenta de análise disponível para contas empresariais dessa rede, ajudando ao administrador da conta a aprender mais sobre seus seguidores por meio de métricas como "alcance" e "impressões".

Ainda, em relação ao público (seguidores), segundo os dados coletados no Instagram $^{\circledR}$, cabe destacar as seguintes cidades alcançadas: Governador Valadares (11,9\%); Juiz de Fora (6,4\%); Ipatinga (6,1\%); São Paulo (4,3\%), sendo os outros $71,3 \%$ dos seguidores pertencentes a outras localidades. Quanto à nacionalidade dos seguidores, 98,8\% destes acessaram o perfil em território brasileiro, $0,6 \%$ em território boliviano, $0,3 \%$ em paraguaio e $0,3 \%$ nos Estados Unidos da América. A faixa etária preponderante se concentrou nos 18 a 24 anos, com 44,3\% dos seguidores, seguido do intervalo entre 25 e 34 anos, com 30,4\%, sendo válido destacar que 3,7\% dos seguidores possuíam mais de 55 anos. O perfil foi acessado majoritariamente por mulheres, correspondendo a $74,3 \%$ dos seguidores totais.

Em relação às publicações, no presente estudo foram realizadas $136 \mathrm{em}$ cada uma das três redes sociais - Instagram ${ }^{\circledR}$, Facebook $^{\circledR}$ e Twitter $^{\circledR}$, que foram compartilhadas até o dia 03/06/2021, sendo 30 teóricas (quadro 1), 44 práticas (quadro 2) e 62 referentes à Covid (quadro 3). As publicações receberam 5230 curtidas no total, conforme descrito nos quadros 1,2 e 3 ,

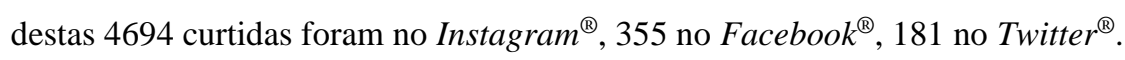

No Quadro 1 são apresentadas 30 publicações, incluindo dois vídeos, com temas teóricos referentes ao SUS e a 
quantidade de curtidas no Instagram $^{\circledR}$ e nas demais redes sociais. Essas publicações foram agrupadas de acordo com a ordem cronológica de postagem, interagindo com o usuário acerca de seus direitos e deveres, sobre legislações especificas, os princípios doutrinários, as políticas públicas, o conselho de saúde e a estrutura do SUS. As referências empregadas na redação dessas postagens foram os sites do Ministério da Saúde (MS), Conselho Nacional de Saúde (CNS), Agência de Vigilância em Saúde (ANVISA) Organização Mundial da Saúde (OMS), Organização Pan Americana de Saúde (OPAS), Conselho Nacional de Secretários de Saúde (CONASS), Conselho Nacional de Secretários Municipais de Saúde (CONASEMS), Sociedade Brasileira de Medicina de Família e Comunidade (SBMFC), Associação Brasileira de Saúde Coletiva (ABRASCO) e Fundação Oswaldo Cruz (FIOCRUZ), revistas cientificas e estudos de universidades nacionais e internacionais como Universidade de São Paulo (USP), Universidade Federal de Pelotas (UFPel), Universidade Federal de Minas Gerais (UFMG), Imperial College, John Hopkins e Oxford, dentre outras.

A quantidade total de curtidas das publicações teóricas em relação ao SUS no Instagram $^{\circledR}$ foi 951, média de 31,7, variando de 15 a 92 curtidas. Nas outras redes, Facebook ${ }^{\circledR}$ mais Twitter $^{\circledR}$, o total de curtidas foi de 132, com média de 4,4, variando de 2 a 8 curtidas. Pode-se observar que no Instagram $^{\circledR}$ o número de curtidas foi sete vezes superior às demais redes sociais, o que nos leva a inferir que esta rede tem um perfil de seguidores mais voltado a este tipo de informação e perfil.

Quadro 1. Publicações com temas teóricos referentes ao SUS e a quantidade de curtidas no Instagram $^{\circledR}$ e no Facebook $^{\circledR}$ mais Twitter $^{\circledR}$, no período compreendido entre julho de 2020 a junho de 2021.

\begin{tabular}{|c|c|c|}
\hline Publicações & $\begin{array}{c}\text { Quantidade } \\
\text { curtidas } \\
\text { Instagram }^{\circledR}(\mathbf{n})\end{array}$ & $\begin{array}{c}\text { Quantidade curtidas } \\
\text { Facebook }^{\circledR}+\text { Twitter }^{\circledR} \text { (n) }\end{array}$ \\
\hline 1. "Você conhece seus direitos e deveres como usuário do SUS" & 39 & 2 \\
\hline 2. "Quais são os serviços ofertados pelo SUS?" & 49 & 6 \\
\hline 3. "Você conhece as leis orgânicas da saúde?" & 38 & 5 \\
\hline 4. "Você conhece a Lei n 8080/1990?" & 25 & 3 \\
\hline 5. "Você conhece a Lei n 8142/1990?" & 36 & 6 \\
\hline 6. "Carta dos Direitos dos Usuários da Saúde: princípios da cidadania" & 27 & 3 \\
\hline 7. "Você conhece a estrutura do SUS?" & 92 & 6 \\
\hline 8.Você conhece os princípios doutrinários e organizativos do SUS? & 34 & 5 \\
\hline 9. "Você conhece as políticas públicas de saúde do SUS?" & 52 & 5 \\
\hline 10. "Políticas de Promoção, Prevenção e Recuperação da Saúde" & 16 & 2 \\
\hline 11. "Política de Controle de Doenças e Enfrentamento de Agravos de Saúde" & 18 & 3 \\
\hline 12. "Políticas Voltadas à Saúde de Segmentos Populacionais" & 20 & 6 \\
\hline 13. "Políticas de Promoção da Equidade em Saúde" & 16 & 5 \\
\hline 14. "Políticas de Organização da Atenção à Saúde" & 21 & 4 \\
\hline 15. "Políticas de Atenção a Agravos Específicos" & 15 & 3 \\
\hline 16. "Políticas de Organização do SUS" & 24 & 6 \\
\hline 17. "RENASES: Relação Nacional de Ações e Serviços de Saúde" & 19 & 5 \\
\hline 18. "RENAME: Relação Nacional de Medicamentos Essenciais" & 18 & 4 \\
\hline 19. "Assistência farmacêutica no SUS" & 28 & 5 \\
\hline 20. "Conselho de saúde" & 24 & 4 \\
\hline 21. "Saúde da população negra" & 20 & 6 \\
\hline 22. "Política de Saúde Mental do SUS" & 48 & 5 \\
\hline 23. "Você conhece a política nacional de humanização?" & 21 & 4 \\
\hline 24. "Vídeo homenagem aos 30 anos do SUS" & 48 & 3 \\
\hline
\end{tabular}




\begin{tabular}{|c|c|c|}
\hline 25. "Infecções sexualmente transmissíveis e HIV/Aids no SUS" & 36 & 5 \\
\hline 26. "Saúde da mulher e da criança no SUS" & 31 & 6 \\
\hline 27. "Saúde indígena no SUS" & 27 & 3 \\
\hline 28. "Programa Saúde na Escola no SUS" & 22 & 3 \\
\hline 29. "Programa Melhor em Casa: assistência domiciliar pelo SUS" & 49 & 5 \\
\hline 30. "Vídeo o SUS é da gente" & 38 & 4 \\
\hline Total & 951 & 132 \\
\hline Média & 31,7 & 4,4 \\
\hline
\end{tabular}

Fonte: Contas do projeto Conecta-SUS no Instagram $^{\circledR}$, Facebook $^{\circledR}$ e Twitter $^{\circledR}$ (2021).

Pelos dados obtidos e pela experiência vivenciada pelos alunos fica evidente que a transmissão de conhecimento digital gera impactos, tanto na população, quanto nos discentes. Essa transmissão de conhecimentos permitiu que a população tenha acesso de forma rápida e acessível, por meio de numa linguagem simples, a várias informações desconhecidas ou não compreendidas sobre o SUS. A atuação dos professores no papel de tutores na condução das informações compartilhadas e sua verificação teve por finalidade garantir que as publicações fossem baseadas sempre na melhor informação disponível sobre os temas e a garantia da utilização de fontes de informações oficiais.

Os impactos na formação dos discentes do Curso de Medicina envolvidos no desenvolvimento desse projeto estão atrelados ao enriquecimento teórico propiciado pela busca e seleção de dados teóricos confiáveis sobre o funcionamento do SUS, a correta e eficiente adaptação desses dados para as redes sociais, buscando sempre uma linguagem compreensível pela população, a qual permite avançar para uma relação médico/paciente mais horizontalizada e, também, ao trabalho em equipe na elaboração do conteúdo, que oferece uma oportunidade de ganho de experiência no convívio profissional.

O Facebook ${ }^{\circledR}{\text {, } \text { Twitter }^{\circledR}, \text { Instagram }^{\circledR} \text { e YouTube }}^{\circledR}$ são mídias reconhecidas internacionalmente como ferramentas para a promoção de projetos de pesquisa, fornecimento e disseminação de informações de saúde e facilitação da educação de estudantes e profissionais (França, Rabello \& Magnago, 2019).

Nessa perspectiva, no contexto do SUS, o médico de família e comunidade é "um especialista em APS, portanto, deve ter formação geral que lhe permita ser o primeiro contato do paciente, sempre que esse procure o serviço de saúde. Nesse contexto o atendimento das pessoas, famílias e comunidade será integral por meio de ações preventivas e terapêuticas" (Barbosa et al, 2021, p.50882).

No Quadro 2 estão apresentadas 44 publicações, incluindo quatro vídeos, postadas nas redes sociais, com temas práticos abordando os serviços disponibilizados pelo SUS.

Quadro 2. Publicações com temas práticos referentes aos serviços disponibilizados pelo SUS a quantidade de curtidas no Instagram $^{\circledR}$ e no Facebook ${ }^{\circledR}$ mais Twitter $^{\circledR}$, no período compreendido entre julho de 2020 a junho de 2021.

\begin{tabular}{|l|c|c|}
\hline \multicolumn{1}{|c|}{ Publicação } & $\begin{array}{c}\text { Quantidade } \\
\text { curtidas } \\
\text { Instagram } \\
\text { (n) }\end{array}$ & $\begin{array}{c}\text { Quantidade curtidas } \\
\text { Facebook } \\
\text { Twitter }\end{array}$ \\
(n)
\end{tabular}


Research, Society and Development, v. 10, n. 11, e111101119434, 2021

(CC BY 4.0) | ISSN 2525-3409 | DOI: http://dx.doi.org/10.33448/rsd-v10i11.19434

\begin{tabular}{|c|c|c|}
\hline 5. "Você já ouviu falar dos aplicativos do SUS? Parte 2" & 23 & 4 \\
\hline 6. "Você já ouviu falar dos aplicativos do SUS? Parte 3" & 30 & 5 \\
\hline 7. "Você já ouviu falar dos aplicativos do SUS? Parte 4" & 17 & 4 \\
\hline 8. "Você já ouviu falar dos aplicativos do SUS? Parte 5" & 22 & 3 \\
\hline 9. "Ouvidoria SUS: Veja como funciona" & 22 & 6 \\
\hline 10. "Telefones úteis do SUS" & 36 & 4 \\
\hline 11. "Você conhece o programa farmácia popular do Brasil?" & 24 & 3 \\
\hline 12. "Cuidados em saúde mental durante a pandemia" & 29 & 3 \\
\hline 13. "Vídeo TeleSUS" & 12 & 4 \\
\hline 14. "Homenagem aos 30 anos do SUS" & 19 & 6 \\
\hline 15. "Vídeo sobre a PEC da Morte" & 51 & 3 \\
\hline 16. "Programa Nacional de Controle do Tabagismo no SUS" & 33 & 6 \\
\hline 17. "Vídeo calendário de vacinação do SUS" & 78 & 2 \\
\hline 18. “Cartão SUS” & 48 & 3 \\
\hline 19. "Programa Nacional de Vacinação do SUS" & 24 & 2 \\
\hline 20. "Manejo da hipertensão arterial no SUS" & 27 & 4 \\
\hline 21. "Manejo da diabetes no SUS" & 36 & 4 \\
\hline 22. "HIV e infecções sexualmente transmissíveis no SUS" & 32 & 5 \\
\hline 23. "Manejo do câncer no SUS" & 34 & 2 \\
\hline 24. "Transplante de órgãos no SUS" & 24 & 5 \\
\hline 25. "Órteses e próteses no SUS" & 31 & 5 \\
\hline 26. "Gravidez e pré-natal no SUS" & 38 & 4 \\
\hline 27. "Saúde e direitos da população LGBTQIA+ no SUS" & 48 & 4 \\
\hline 28. "Vídeo defenda o SUS" & 28 & 3 \\
\hline 29. "Saúde da pessoa idosa no SUS" & 45 & 4 \\
\hline 30. "Saúde do jovem e do adolescente no SUS" & 34 & 3 \\
\hline 31. "Saúde do homem no SUS" & 29 & 2 \\
\hline 32. "Serviço de atendimento móvel de urgência (SAMU) do SUS" & 35 & 4 \\
\hline 33. "Medicamentos excepcionais oferecidos pelo SUS" & 50 & 4 \\
\hline 34. "Doenças raras no SUS" & 34 & 4 \\
\hline 35. "Saúde mental no SUS" & 43 & 4 \\
\hline 36. "Atendimento odontológico no SUS" & 42 & 4 \\
\hline 37. "Violência sexual no âmbito do SUS" & 45 & 4 \\
\hline 38. "Doenças pulmonares no SUS" & 28 & 4 \\
\hline
\end{tabular}




\begin{tabular}{|l|c|c|}
\hline 39. "Telessaúde no SUS" & 27 & 3 \\
\hline 40. "Saúde na hora" & 37 & 4 \\
\hline 41. "Manejo da gripe no SUS" & 19 & 4 \\
\hline 42. "Manejo da dengue no SUS" & 29 & 4 \\
\hline 43. "Manejo da Zika e da Chikungunya no SUS" & 27 & 3 \\
\hline 44. "Manejo da hanseníase no SUS" & 23 & 3 \\
\hline Total & 1428 & 172 \\
\hline Média & 33,21 & 3,9 \\
\hline
\end{tabular}

Fonte: Contas do projeto Conecta-SUS no Instagram $^{\circledR}$, Facebook $^{\circledR}$ e Twitter $^{\circledR}$ (2021).

O maior número de curtidas das postagens de conteúdo prático do SUS foi no Instagram $^{\circledR}$, sendo oito vezes superior às demais redes, variando de 12 a 103 curtidas, com média de 37,38. No Facebook $^{\circledR}$ e Twitter ${ }^{\circledR}$, a média de curtidas foi de 3,7 , variando de 2 a 8 curtidas.

Também foi observado pelos discentes que as publicações de caráter mais prático, envolvendo temas de maior visualização e preocupação social no momento atual, como saúde mental, funcionamento da APS e vacinação, atingiram maiores índices de salvamento e compartilhamento nas publicações, demonstrando maior interesse por parte dos seguidores. Essa constatação vai ao encontro dos achados de Massarani, Waltz \& Leal (2020) ao debaterem sobre pesquisas na área da saúde nas redes sociais, referentes às vacinas, tema que despertou grande interesse dos usuários, com o maior volume de links comentados, curtidos e compartilhados.

Além disso, os temas práticos referentes ao SUS contemplam para o futuro exercício profissional do médico, as áreas de formação, dada a necessária articulação entre conhecimentos, habilidades e atitudes requeridas do egresso, segundo as diretrizes curriculares do Curso de Medicina: I - atenção à Saúde; II - gestão em Saúde; e III - educação em Saúde (Brasil, 2014).

Segundo Bonifácio e Amorim (2020), desde o início do curso de graduação em medicina, a introdução dos estudantes no SUS, pode constituir uma oportunidade para o reforço dos valores éticos que essa profissão requer. Nesse sentido, os discentes desse curso devem "precocemente ser inseridos em práticas relevantes", com seu poder de autonomia, integrar ensino e serviço à formação acadêmica, atendendo às necessidades sociais da saúde com priorização do SUS (Vasconcelos \& Ruiz, 2015).

No Quadro 3 são apresentadas 62 publicações, incluindo 13 vídeos, postadas nas redes sociais, com temas relacionados à pandemia da Covid-19. As postagens foram elaboradas tendo como referência as publicações dos sites do MS, CONASS, CONASEMS, CNS, ONU Brasil, ANVISA, FIOCRUZ, ABRASCO, CNN Brasil, REDSAUDE, UFJF, UFPel, UFMG, Pan American Health Organization (PAHO), Public Medline (PUBMED), Fundação Nacional de Saúde (FUNASA), World Organization Health (WHO), British Broadcasting Corporation (BBC), Pontifícia Universidade Católica do Rio Grande do Sul (PUCRS), Laboratório Pfizer, National Geographic, Governo de Minas Gerais, São Paulo e Espírito Santo, Instituto Butantan, Consórcio de Veículos de Imprensa, Jornal El Pais, Senado Federal do Brasil, Sociedade Brasileira de Infectologia e Agência Brasil. 
Quadro 3. Publicações com temas referentes à Covid-19 e a quantidade de curtidas no Instagram $^{\circledR}$ e no Facebook $^{\circledR}$ mais Twitter $^{\circledR}$, no período compreendido entre julho de 2020 a junho de 2021.

\begin{tabular}{|c|c|c|}
\hline Publicação & $\begin{array}{l}\text { Quantidade } \\
\text { curtidas } \\
\text { Instagram }^{\circledR} \\
\text { (n) }\end{array}$ & $\begin{array}{l}\text { Quantidade } \\
\text { curtidas } \\
\text { Facebook }^{\circledR}+ \\
\text { Twitter }^{\circledR} \\
\text { (n) }\end{array}$ \\
\hline 1. "Aprovação de indenização para profissionais da saúde afetados pela Covid" & 34 & 7 \\
\hline 2. "Linha do Tempo Coronavírus" & 29 & 2 \\
\hline 3. "Confira a seguir alguns mitos e verdades sobre a COVID-19" & 63 & 5 \\
\hline 4. "Vídeo do CNS sobre Covid e Hidroxicloroquina" & 82 & 5 \\
\hline 5. "Vamos falar sobre medicamentos e a Covid-19?" & 47 & 6 \\
\hline 6. "Vídeo da@onubrasil em apoio ao uso de máscaras" & 103 & 4 \\
\hline 7. "Quais são os testes disponíveis para a Covid-19?" & 36 & 3 \\
\hline 8. "Vídeo sobre a Covid-19 da @onubrasil” & 57 & 5 \\
\hline 9. "Quais são os principais sintomas da Covid-19?" & 38 & 3 \\
\hline 10. "Como os profissionais de saúde podem se proteger em tempos de pandemia?" & 20 & 4 \\
\hline 11. "Vídeo sobre a marcha pela vida" & 92 & 6 \\
\hline 12. "Vídeo sobre reabertura das escolas da @onubrasil" & 28 & 3 \\
\hline 13. "Vídeo sobre o desafio use máscara" & 21 & 3 \\
\hline 14. "Vacinação e Covid-19" & 21 & 4 \\
\hline 15. "Vídeo higienização das mãos @onubrasil" & 35 & 4 \\
\hline 16. "Vídeo da UFJF em apoio ao movimento use máscara" & 31 & 3 \\
\hline 17. "Falando sobre as máscaras" & 38 & 2 \\
\hline 18. "Vídeo sobre isolamento domiciliar do Ministério da Saúde" & 46 & 2 \\
\hline 19. "A importância do isolamento social em tempos de pandemia" & 26 & 5 \\
\hline 20. "4 formas de se consultar pelo SUS sem sair de casa nessa pandemia" & 32 & 5 \\
\hline 21. "Vídeo sobre saúde mental na pandemia" & 40 & 4 \\
\hline 22. "Como se proteger do coronavírus ao ir às compras?" & 24 & 3 \\
\hline 23. "Doação de sangue: o que mudou com a pandemia do novo coronavírus?" & 31 & 7 \\
\hline 24. "Impactos econômicos da Covid-19 na primeira infância" & 31 & 4 \\
\hline 25. "Dúvidas frequentes relacionadas à Covid-19" & 38 & 3 \\
\hline 26. "Idosos e saúde mental em tempos de pandemia" & 24 & 5 \\
\hline 27. "Vídeo sobre imunidade de rebanho" & 94 & 4 \\
\hline 28. "Dicas para organizar o trabalho e o estudo remoto na pandemia" & 25 & 2 \\
\hline 29. "Covid-19 e a transmissão pelo ar" & 17 & 2 \\
\hline 30. "Vídeo sobre a importância da solidariedade em tempos de pandemia" & 32 & 1 \\
\hline 31. "Segunda onda da pandemia de Covid -19" & 62 & 5 \\
\hline 32. "A importância do SUS no combate à Covid-19" & 22 & 3 \\
\hline 33. "Risco de transmissão da Covid-19 em diferentes situações" & 25 & 3 \\
\hline 34. "Vídeo sobre o que se fazer se alguém da residência apresentar sintomas da Covid-19" & 39 & 2 \\
\hline 35. "Atualizações sobre a Covid-19" & 16 & 3 \\
\hline 36. "Síndrome pós-Covid-19" & 21 & 2 \\
\hline 37. “Gestação e Covid-19” & 37 & 4 \\
\hline 38. "Crianças e a Covid-19" & 26 & 3 \\
\hline 39. "Compilado geral das vacinas em tabela" & 19 & 3 \\
\hline 40. "Hipertensão e diabetes durante a pandemia" & 22 & 4 \\
\hline 41. "Hipóxia silenciosa e a Covid-19" & 39 & 6 \\
\hline
\end{tabular}




\begin{tabular}{|c|c|c|}
\hline 42. "Vacinas contra Covid-19" & 46 & 5 \\
\hline 43. "Motivos para se vacinar contra a Covid-19" & 50 & 6 \\
\hline 44. "Dúvidas sobre a vacina Coronavac" & 47 & 3 \\
\hline 45. "O que o SUS vem fazendo para enfrentar a pandemia de Covid-19" & 43 & 4 \\
\hline 46. "A pandemia da Covid-19 e a desigualdade social" & 34 & 3 \\
\hline 47. "Como saber se a vacina da Covid-19 foi aplicada corretamente?" & 44 & 3 \\
\hline 48. "Novas variantes do coronavírus pelo mundo" & 45 & 3 \\
\hline 49. "Impactos da vacinação contra Covid-19 no SUS" & 34 & 5 \\
\hline 50. "Vacinas eficazes contra as novas variantes do coronavírus" & 39 & 3 \\
\hline 51. "Planos de vacinação do SUS contra a Covid-19" & 27 & 3 \\
\hline 52. "Planos de classificação de risco para Covid-19 no Sudeste" & 25 & 3 \\
\hline 53. "Vacinação contra Covid-19 em crianças e adolescentes" & 30 & 5 \\
\hline 54. "Atualização do uso de máscaras na pandemia da Covid-19" & 43 & 4 \\
\hline 55. "Vídeo infectologista sobre descontrole da Covid-19" & 75 & 2 \\
\hline 56. "Tempo de imunidade contra Covid-19" & 24 & 3 \\
\hline 57. "Atualização sobre as vacinas contra a Covid-19" & 31 & 3 \\
\hline 58. "Rede de urgência e emergência do SUS na pandemia da Covid-19" & 15 & 4 \\
\hline 59. "Impacto da vacinação contra Covid-19 em idosos e profissionais da saúde no Brasil" & 36 & 5 \\
\hline 60. "Dúvidas sobre a vacina da Pfizer contra a Covid-19" & 25 & 3 \\
\hline 61. "As comorbidades e a vacinação contra Covid-19" & 21 & 4 \\
\hline 62. "Variante indiana do novo coronavírus" & 18 & 4 \\
\hline Total & 2315 & 232 \\
\hline Média & 37,38 & 3,74 \\
\hline
\end{tabular}

Fonte: Contas do projeto Conecta-SUS no Instagram $^{\circledR}$, Facebook $^{\circledR}$ e Twitter $^{\circledR}$ (2021)

O maior número de curtidas foi no Instagram $^{\circledR}$, sendo dez vezes superior às demais redes sociais, variando de 12 a 47 curtidas, com média de 33,21. No Facebook $^{\circledR}$ e Twitter $^{\circledR}$, a média de curtidas foi de 3,9, variando de 2 a 8 curtidas.

A Covid-19 é um tema de interesse das pessoas e as postagens são feitas a todo instante nas redes sociais, plataformas com alta velocidade de geração de dados. Nesse sentido o estudo de Xavier et al (2020) mostra que foram coletadas no Twitter mais de 7,7 milhões de postagens em português relacionadas à Covid-19, em 62 dias de coleta, resultando em média cerca de 130 mil postagens por dia ou 5.188 postagens por hora.

No enfrentamento da pandemia da Covid-19 a APS é a porta preferencial de acesso às várias interfaces do SUS e suas ações comunitárias. Essa doença vem exigindo uma remodelagem de estruturas, não somente na assistência hospitalar, mas, sobretudo nas intervenções nas comunidades e seus problemas sociais, urbanos e estruturais. Destaca-se o processo de trabalho e as novas incorporações técnicas e dinâmicas para prevenção, vigilância e monitoramento impostas pelo Sars-CoV2 à APS que deve, ainda, garantir o acesso aos cuidados essenciais. A APS é apresentada como uma força fundamental na proteção e promoção da saúde dos indivíduos, famílias e comunidade envolvida na resposta de curto, médio e longo prazo à Covid-19 (Silva et al, 2021). É importante destacar também que o SUS, um sistema de saúde com cobertura universal no contexto da pandemia

"será sempre a opção mais humanizada e mais digna de acesso a saúde, pois tem como princípio doutrinário a cobertura estendida a todos os cidadãos, geralmente se organiza a partir de um nível assistencial denominado APS, onde se garante de forma ampla e aberta acesso para todas as pessoas aos serviços e ações de promoção e proteção à saúde, e de prevenção de doenças, tão essenciais para o bem-estar do homem e para o desenvolvimento económico e social sustentável de um país" (Barbosa, 2020, p.1)

As redes sociais como fontes de informação estão se tornando imperativos na sociedade brasileira cada vez mais 
conectada, de modo que as pessoas formam uma rede com potencial para disseminar prontamente suas próprias interpretações de informações sobre saúde pública, fora de um contexto ou estrutura científica, e sem conteúdo de fontes seguras e validadas. As redes sociais da web estão disponíveis gratuitamente, são fáceis de usar, não têm supervisão por parte das autoridades e podem promover um ambiente de informações com fontes seguras, cientificas e oficiais, de órgãos governamentais para que não se apresentem de forma tendenciosa ou errôneas, podendo causar agravos à saúde individual ou da comunidade (Khan et al., 2010).

Ressalta-se que há uma necessidade significativa de adquirir uma cultura de saúde pública que esteja aberta ao compartilhamento seguro e oportuno de informações. Para lidar com as barreiras culturais ao compartilhamento de informações, qualquer inovação da informação no campo da saúde pública deve agregar valor a todos os componentes de um sistema de saúde, garantindo acesso à informação de qualidade em tempo oportuno a toda a nação. Para garantir esse acesso, devemos melhorar coletivamente nossas capacidades de detecção, alerta e previsão de doenças. A revolução da internet, o aumento da disponibilidade de informações eletrônicas relacionadas à saúde e a melhoria da tecnologia da informação deram um acesso sem precedentes a novos fluxos de informação e a capacidade de estabelecer redes sociais para divulgação de ações e serviços de saúde, para prevenção de agravos e doenças e para promoção de saúde das populações (Khan et al. 2010).

\section{Considerações Finais}

Diante do cenário pandêmico e as novas realidades sociais que transformaram o setor da comunicação, aumentando a utilização das redes sociais e, com isso, a circulação mais frequente de notícias falsas, o projeto Conecta-SUS desempenha a considerável função de divulgar informações precisas e objetivas sobre o SUS, esclarecendo dúvidas e conscientizando os cidadãos sobre seus direitos como usuários deste sistema, para que eles desenvolvam maior protagonismo e autonomia acerca de seu processo saúde/doença e sobre a rede de serviços de atenção à saúde pública e seus parceiros complementares.

Além disso, o projeto acumula contribuição no esclarecimento à população sobre o período pandêmico, divulgando os fatos mais importantes que acontecem durante esse contexto, baseando-se em fontes formais e seguras, além de trazer aos alunos extensionistas uma compreensão mais profunda sobre todo o funcionamento e os serviços ofertados pelo SUS durante a pandemia na medida em que geram conteúdos para as publicações nos perfis do projeto nas redes sociais.

Diante das métricas obtidas, percebe-se que a página do Conecta-SUS alcançou um abrangente número de pessoas e gerou um amplo engajamento do público, mostrando que a ferramenta digital é um meio indispensável de capilarização das informações, alcançando a população de forma eficiente e acessível.

\section{Referências}

Barbosa, A. F. (2014). TIC Saúde 2014: [livro eletrônico]: pesquisa sobre o uso das tecnologias de informação e comunicação nos estabelecimentos de saúde brasileiros, (2a ed.), Comitê Gestor da Internet no Brasil.

Barbosa. S. P. (2020). A atenção primária à saúde no contexto da COVID-19. HU Rev. 46:1-2. http://dx.doi.org/10.34019/1982-8047.

Barbosa, S. P.; Paula, P. A. B., Amorim, M. A. A, Cavalcante, R.B., Oliveira, Y., Serejo, J. J., \& Pio, L. M. O. (2021). A medicina de família e comunidade na perspectiva dos discentes e docentes das universidades públicas do estado de Minas Gerais. Brazilian Journal of Development, 7(5), 50879-50896. http://dx.doi.org/10.34117/bjdv7n5-471.

Brasil (1990). Lei $n^{\circ} 8.080$, de 19 de setembro de 1990. Lei Orgânica da Saúde. Dispõe sobre as condições para a promoção, proteção e recuperação da saúde, a organização e o funcionamento dos serviços correspondentes e dá outras providências. Brasília. http://conselho.saude.gov.br/ legislacao/lei8080_190990.htm.

Brasil (2014). Ministério da Educação. Diretrizes nacionais do curso de medicina. 2014. http://portal.mec.gov.br/index.php?opti on=com_docman\&view=download\&alias=1587 4-rces003-14\&category_slug=junho-2014-pdf\&Itemid=30192.

Brasil (2018). Núcleo de informação e coordenação do Ponto BR. Pesquisa sobre o uso das tecnologias de informação e comunicação nos estabelecimentos de saúde brasileiros: TIC saúde 2017, São Paulo, Comitê Gestor da Internet no Brasil, 2018. https://www.nic.br/media/docs/publicacoes/2/tic_saude_2017_livro_eletronico.pdf 
Research, Society and Development, v. 10, n. 11, e111101119434, 2021

(CC BY 4.0) | ISSN 2525-3409 | DOI: http://dx.doi.org/10.33448/rsd-v10i11.19434

Bonifácio, U. A., \& Amorim, M. M. A. (2020). Projeto de intervenção na educação permanente para os médicos da unidade básica de saúde Jardim Brasília, Uberlândia - Minas Gerais. Revista Gepesvida, 11(5), 63-77. http://www.icepsc.com.br/ojs/index.php/gepesvida/article/view/349/179:

França, T, Rabello, E. R., \& Magnago, C. (2019). As mídias e as plataformas digitais no campo da Educação Permanente em Saúde: debates e propostas. Saúde debate 43 (spe1), 106-115. https://doi.org/10.1590/0103-11042019S109.

Khan, A. S., Fleischauer, A. Casani, J., \& Groseclose, S. L. (2010). The Next Public Health Revolution: Public Health Information Fusion and Social Networks. American Journal of Public Health. Disponível em https://ajph.aphapublications.org/doi/full/10.2105/AJPH.2009.180489. Acesso em: 10 ago. 2021

Marteleto, RM (2010). Redes sociais, mediação e apropriação de informações: situando campos, objetos e conceitos na pesquisa em Ciência da Informação, Pesquisa Brasileira em Ciência da Informação e Biblioteconomia, 3(1), 27-46. https://www.arca.fiocruz.br/bitstream/icict/2247/1/Mar teleto_redes\%20sociais\%20mediacao\%20e\%20apropriacao\%20de\%20informacoes.pdf.

Massarani L, Waltz, I., \& Leal, T. (2020). O debate sobre vacinas em redes sociais: uma análise exploratória dos links com maior engajamento. Cad. Saúde Pública 36 (Suppl 2), e00148319. https://doi.org/10.1590/0102-311X00148319.

Moretti, F. A., Oliveira, V. E., \& Silva, E. M. K (2012). Acesso a informações de saúde na internet: uma questão de saúde pública? Rev. Assoc. Med. Bras., 58 (6), 650-658. http://www.scielo.br/scielo.php?script=sci_arttext\&pid=S0104- 42302012000600008\&lng=en\&nrm=isso.

OMS (2017). Organização Mundial da Saúde. 67 Health Intervention and Technology Assessment in Support of Universal Health Coverage. Assembléia Mundial de Saúde, 67(23), 1-10. http://apps.who.int/medicinedocs/documents/s21463en/s21463en.pdf.

Silva, A.V. F. G., Barbosa, S. P., Lanza, F. M., Amorim, M. M. A., \& Mássimo, E. A. L. T. (2021). The COVID-19 in the Primary Health Care contexto. Research, Society and Development, 10(3), e49010313602. http://dx.doi.org/10.33448/rsd-v10i3.13602.

Vasconcelos, R. N. C., \& Ruiz, E. M. (2015). Formação de Médicos para o SUS: a Integração Ensino e Saúde da Família: Revisão Integrativa, Revista Brasileira de Educação Médica (Online), 39(4), 630-638. https://doi.org/10.1590/1981-52712015v39n4e02772014.

Xavier, F, Olensckiandre, J. R. W, Acosta, A. L., Sallum, M. A. M., \& Saraiva A. M. Análise de redes sociais como estratégia de apoio à vigilância em saúde durante a Covid-19. Estudos Avançados, 34 (99), 2020. https://doi.org/10.1590/s0103-4014.2020.3499.016. 